\title{
Region-based Weighted Prediction Algorithm for H.264/AVC Video Coding
}

\author{
Sik-Ho Tsang, Tsz-Kwan Lee, Yui-Lam Chan and Wan-Chi Siu \\ Centre for Signal Processing, Department of Electronic and Information Engineering \\ The Hong Kong Polytechnic University \\ Hung Hom, Hong Kong \\ Email: \{en.ho, eie.glorylee\}@connect.polyu.hk, \{enylchan, enwcsiu\}@polyu.edu.hk
}

\begin{abstract}
This paper proposes a novel region-based weighted prediction (WP) algorithm to encode scenes with complex brightness variations. It facilitates the use of multiple WP parameter sets in a single reference frame by utilizing the framework of multiple reference frame motion estimation (MRF-ME). With this arrangement, different macroblocks in the current frame can use different WP parameter sets even when they are predicted from the same reference frame. To support this, a region partitioning process is designed to divide the current frame into different regions where each one has some degree of uniformity in its brightness variation. Multiple sets of region-based WP parameters can then be estimated accurately. Consequently, the proposed algorithm can improve prediction in scenes with different degrees of brightness variations in different regions of the same picture. Results show that the region-based algorithm can achieve significant coding gains of scenes with complex brightness variations.
\end{abstract}

\section{INTRODUCTION}

Block-based motion estimation and compensation play an indispensible role to reduce the temporal redundancy in video coding by minimizing the prediction error between frames. It assumes brightness level between frames is constant, and changes between frames are caused by object movements or camera motions only. Whenever there is brightness variation between frames, the coding efficiency may be reduced. To handle videos with brightness variation such as fade in/out effects, the H.264/AVC standard [1] introduced a weighted prediction (WP) tool [2] to enhance motion compensation with a WP parameter set which includes a weighting factor $W_{i}$ and an offset $O_{i}$. $\quad W_{i}$ and $O_{i}$ are involved to estimate the sum of absolute differences (SAD) during motion estimation between the block of the current frame $F_{\text {curr }}, B_{\text {curr }}$, and the block of the $\mathrm{i}^{\text {th }}$ reference frame $F_{i}, B_{i}$, as follows:

$$
S A D\left(B_{\text {curr }}, B_{i}\right)=\sum_{P_{\text {curr }} \in B_{\text {curr }}, P_{i} \in B_{i}}\left|P_{\text {curr }}-\left(P_{i} \cdot W_{i} / W_{D}+O_{i}\right)\right|(1)
$$

where $P_{\text {curr }}$ and $P_{i}$ are the pixel values within $B_{\text {curr }}$ and $B_{i}$ respectively, and $W_{D}$ is to provide the granularity for $W_{i}$. In
H.264, a LMS model is used [3] to determine the WP parameter set:

$$
\begin{aligned}
W_{i} & =W_{D} \cdot \sum_{P_{\text {cur }} \in F_{\text {cur }}}\left|P_{\text {curr }}-\overline{P_{\text {curr }}}\right| / \sum_{P_{i} \in F_{i}}\left|P_{i}-\overline{P_{i}}\right| \\
O_{i} & =\overline{P_{\text {curr }}}-\overline{P_{i}} \cdot W_{i} / W_{D}
\end{aligned}
$$

where $\overline{P_{\text {curr }}}$ and $\bar{P}_{i}$ are the mean value of $P_{\text {curr }}$ and $P_{i}$. However this frame-based weighted prediction (FWP) approach can handle sequences with global brightness variation (GBV) only since the WP parameter set is determined in frame basis. To tackle the problem of local brightness variation (LBV), macroblock(MB)-based scheme [4], where each MB has its own WP parameter set, was proposed. However, it results in excessive overhead to code a large amount of WP parameter sets. To avoid this, [5] then proposed to estimate the offset for each MB using neighbouring pixels. But irrelevant and noisy pixels always reduce the accuracy of the estimated offset. Besides, both schemes in [4-5] are not compliant to H.264/AVC standard. In this paper, we propose a novel region-based WP parameter estimation algorithm for encoders of the H.264/MPEG-4 AVC standard. It can handle complex LBV scenes with more than one region.

\section{PRoposed REgION-BASED WP (RWP) SCHEME}

The proposed scheme is particularly designed for coding scenes with LBV. It is accomplished by making use of multiple WP parameter sets for various regions and assigning them to the same reference frame. It mainly consists of three steps: (1) region partitioning with a simple WP parameter estimator, (2) accurate estimation of region-based WP parameter sets, and (3) adoption of MRF architecture in WP that supports the coding of multiple WP parameter sets.

\section{A. Region Partitioning}

In the first step, MBs are grouped into different regions based on the degrees of brightness variations. The region partitioning employs a simple WP parameter estimator. For instance, a DC model is used for the computation of $W_{0}\left(M B_{n}\right)$

The work described in this paper is partially supported by the Centre for Signal Processing and a grant from the Internal Competitive Research Grant, Department of EIE, PolyU (PolyU G-YL20). 
which is computed as the ratio of the mean value of $\mathrm{MB}_{\mathrm{n}}$ in $F_{\text {curr }}, \overline{P_{\text {cur }}\left(M B_{n}\right)}$, to the mean value of co-located $\mathrm{MB}, \overline{P_{0}\left(M B_{n}\right)}$, in the nearest reference frame $F_{0}$, given by

$$
W_{0}\left(M B_{n}\right)=W_{D} \cdot\left(\overline{P_{\text {curr }}\left(M B_{n}\right)} / \overline{P_{0}\left(M B_{n}\right)}\right)
$$

Noted that $O_{i}\left(M B_{n}\right)$ is set to zero in this step. Fig. 1(a) and Fig. 1(b) depict the brightness variation histograms using $W_{0}\left(M B_{n}\right)$ for the frames with GBV and $\mathrm{LBV}$, respectively. The histogram is a representation of the distribution of $W_{0}\left(M B_{n}\right)$. It can be observed that the histogram of LBV has a broader spectrum than that of GBV. This is the reason why conventional WP cannot handle scenes with LBV efficiently since only one WP parameter set is not enough to model wide range of brightness variations. Region partitioning in the proposed scheme is to divide $F_{\text {curr }}$ into different regions where each one has some degree of uniformity in its brightness variation. If the possible values of $W_{0}\left(M B_{n}\right)$ are sufficiently small, then each of those may be placed on a range by itself. From Fig. 1(b), it can be seen that the possible values of $W_{0}\left(M B_{n}\right)$ for LBV scenes is too large. It is inefficient to encode too many possible values of $W_{0}\left(M B_{n}\right)$. To avoid this, $W_{0}\left(M B_{n}\right)$ is quantized uniformly by a quantization factor $Q$ into $Q W_{0}\left(M B_{n}\right)$. It then forms an $N$-bin-histogram, each bin containing many similar values of $W_{0}\left(M B_{n}\right)$. Nevertheless, the percentages of MBs in some histogram bins are insignificant, and are considered as unreliable bins. To remove these unreliable bins, only the representative regions with $N_{R}$ largest areas are selected among $N$ regions. After this process, $N_{R}$ regions (denoted as $R_{k}$, where $k=1, \ldots, N_{R}$ ) are formed for the determination of region-based WP parameter sets in the next step.

\section{B. Estimation of Region-based Parameter Sets}

In the second step, instead of using the simple DC model, a quasi-optimal estimator using the LMS model in (2) is adopted to compute the WP parameter sets for $R_{k}$. For each $R_{k}$, (2) can then be modified in region-basis, and written by

$$
\begin{aligned}
& W_{0}\left(R_{k}\right)=W_{D} \cdot \frac{\sum_{P_{\text {curr }} \in F_{\text {curr }}\left(R_{k}\right)}\left|P_{\text {curr }}-\overline{P_{\text {curr }}\left(R_{k}\right)}\right|}{\sum_{P_{0} \in F_{0}\left(R_{k}\right)}\left|P_{0}-\overline{P_{0}\left(R_{k}\right)}\right|} \\
& O_{0}\left(R_{k}\right)=\overline{P_{\text {curr }}\left(R_{k}\right)}-\overline{P_{0}\left(R_{k}\right)} \cdot W_{0}\left(R_{k}\right) / W_{D}
\end{aligned}
$$

where $k=1,2, \ldots, N_{R}$, and $\overline{P_{\text {curr }}\left(R_{k}\right)}$ and $\overline{P_{0}\left(R_{k}\right)}$ are the mean values of the region $R_{k}$ of $F_{\text {curr }}$ and $F_{0}$ respectively. Thereby, $N_{R}$ region-based WP parameter sets, $\left\{W_{0}\left(R_{k}\right), O_{0}\left(R_{k}\right)\right\}$, are estimated. As only relevant pixels are involved to estimate each degree of brightness variation, the estimated WP parameter sets are more accurate than the one estimated in frame basis by FWP as formulated in (2).

\section{Coding of WP Parameter Sets into MRF-ME of H.264}

Normally, in the MRF-ME framework of H.264/AVC, reference frames are listed in a default order from the nearest reference frame assigned with the smallest reference index (ref_idx) to the farthest reference frame assigned with the largest refidx. In conventional FWP scheme, only one single WP parameter set is associated with one ref_idx. In fact, reference frames can be reordered in different kinds of order by signaling few bits in the slice header. One single reference frame can then be associated with more than one WP parameter sets. Interested readers are encouraged to read [67]. Based on this, the region-based WP parameter sets $\left\{W_{0}\left(R_{k}\right), O_{0}\left(R_{k}\right)\right\}$ are encoded using the framework of MRF$\mathrm{ME}$ in H.264. Fig. 2 shows the new arrangement for RWP. Different region-based WP parameter sets, $\left\{W_{0}\left(R_{k}\right), O_{0}\left(R_{k}\right)\right\}$ are applied only to $F_{0}$. Each $\left\{W_{0}\left(R_{k}\right), O_{0}\left(R_{k}\right)\right\}$ represents one single LBV within a certain region in the same reference frame, $F_{0}$. The weighted reference frames associated with different value pairs of $\left\{W_{0}\left(R_{k}\right), O_{0}\left(R_{k}\right)\right\}$ as shown in Fig. 2 are stored in the multiple frame buffers for motion estimation and compensation. This arrangement allows different $\mathrm{MBs}$ in the current frame to employ different value pairs of $\left\{W_{0}\left(R_{k}\right)\right.$, $O_{0}\left(R_{k}\right)$ \}even they are predicted from the same reference frame. By doing so, ref_idx can be used to indicate which $\left\{W_{0}\left(R_{k}\right), O_{0}\left(R_{k}\right)\right\}$ to be used for each MB. It is pointed out that the original $F_{0}$ is still maintained in reference frame buffer for regions without any brightness changes, i.e. weighting factor equals to $W_{D}$ and offset equals to zero.

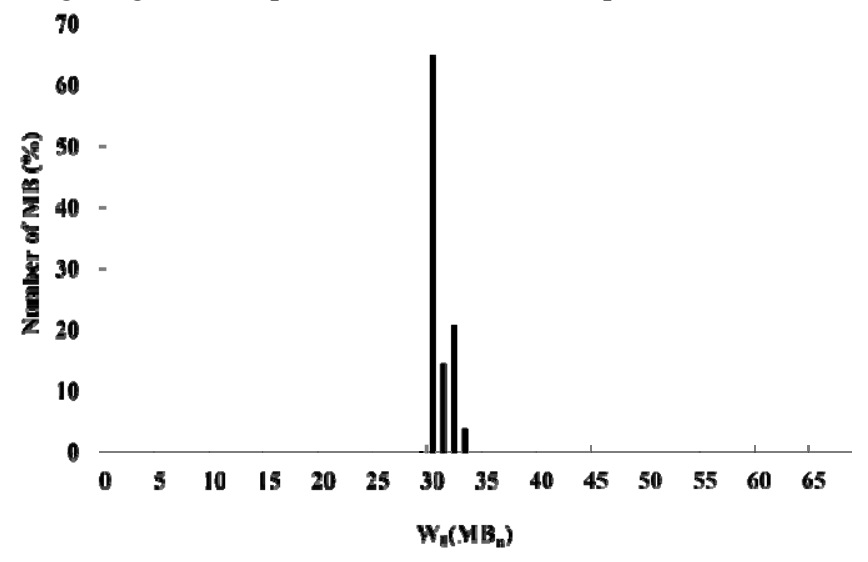

(a)

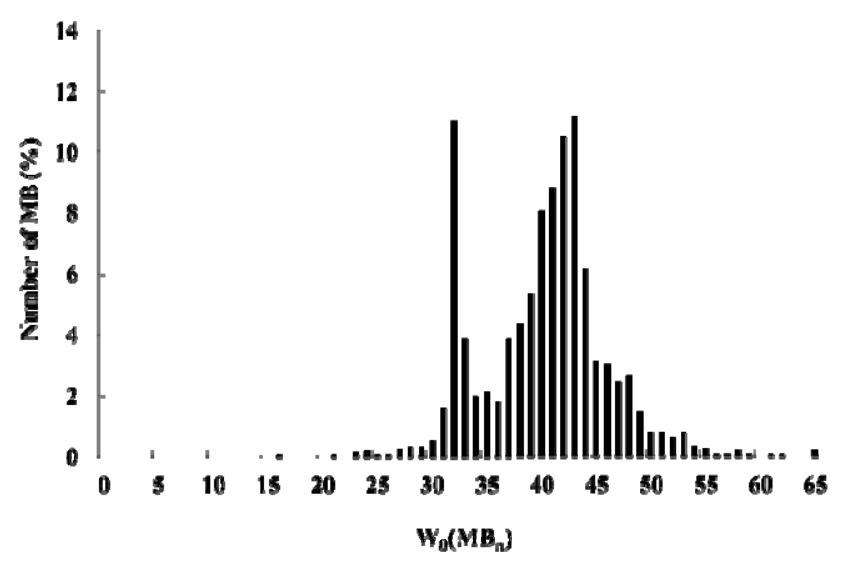

(b)

Figure 1. Histograms for the frames (a) with GBV, and (b) with LBV. 


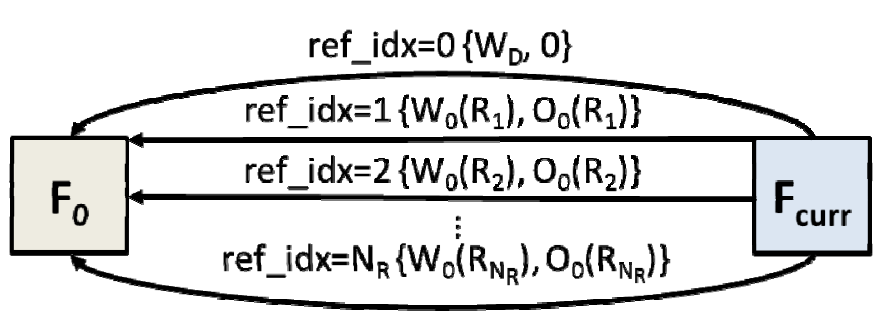

Figure 2. Illustration of RWP with reference reordering for MRF-ME.

\section{Fast RWP}

The proposed RWP is very efficient in compensating different degrees of brightness variation. However, RWP suffers from high computational complexity since it requires performing motion estimation in multiple reference frames, as shown in Fig. 2. It demands more computational complexity as the number of regions, $N_{R}$, increases. To mitigate the complexity, redundant motion estimation process on reference frames that have irrelevant WP parameter sets can be eliminated. Consequently, two fast techniques for RWP including frame-based small region removal (SRR) and MBbased fast reference selection (FRS) are proposed.

\section{1) Frame-based small region removal (SRR)}

In Section II.A, the histogram based on $Q W_{0}\left(M B_{n}\right)$ is generated and only the $N_{R}$ highest bins is used to estimate the region-based WP parameter sets. However, some bins are not crucial for compensating the brightness change since the area of that particular brightness variation is tiny or they are unreliable bins induced by fast object motions or camera movements only. Thus a thresholding technique is applied into the $Q W_{0}\left(M B_{n}\right)$ histogram. If the histogram bins are smaller than a certain threshold $T H$, it is set to zero and not used for estimating the region-based WP parameter sets. As a result, $N_{R}$ becomes smaller, resulting in speeding up the coding process.

\section{2) MB-based fast reference selection (FRS)}

In the first step of RWP, $Q W_{0}\left(M B_{n}\right)$ is formed for region partitioning. The proposed fast algorithm taking into consideration that the brightness variations of the current MB being coded and its adjacent MBs tend to have high correlation. This property could allow us for speeding up the selection of region-based WP parameter sets. Based on $Q W_{0}\left(M B_{n}\right)$ of the current $\mathrm{MB}, Q W_{0}\left(M B_{\text {curr }}\right)$, and those of neighbouring $\mathrm{MBs}$, we can probably select the correct reference index for the current MB before MRF-ME. Fig. 3 shows the idea of FRS. If $Q W_{0}\left(M B_{\text {curr }}\right)$ and all of its neighbouring MBs, as depicted in Fig. 3, are the same, we can consider the particular WP parameter set for the current MB is estimated from only one single reference frame, which is the same as its neighbouring MBs. Motion estimation on other reference frames with inappropriate WP parameter sets can be skipped.

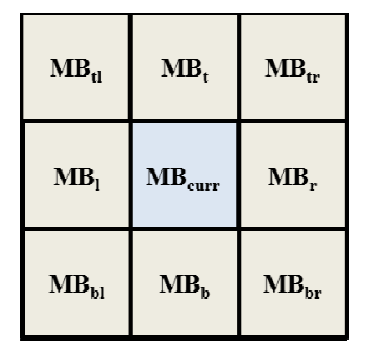

Figure 3. $\mathrm{MB}_{\text {curr }}$ and its neigbouring $\mathrm{MBs}$.

\section{EXPERIMENTAL RESULTS}

We performed computer simulation on two standard video sequences in WVGA format $(832 \times 480)$ - "Mobisode1" and "Mobisode2", which contain plentiful scenes with different types of brightness variations. These two sequences were split into various segments for performance evaluation in either GBV or LBV scenes. The details of those video segments are tabulated in Table I. We have incorporated RWP from Section II.A to Section II.C, and RWP with fast techniques (FastRWP) described in Section II.D, into the latest version of H.264/AVC JM 18.4 reference software [8]. For both RWP and FastRWP, $Q$ and $N_{R}$ in Section II were both set to 4 , i.e. four regions are defined. And $T H$ is set to one-eighth area of the whole frame for FastRWP. They were used to compare the performances of the conventional frame-based WP scheme (FWP) where WP parameter sets are calculated using (2) $[3,8]$ as well as H.264/AVC without any use of WP (NoWP). In addition, all experiments were conducted using $\mathrm{IbBbBbBbP}$ hierarchical-B encoding structure, Main profile, quarter-pel full search motion estimation with search range of \pm 32 pixels, rate-distortion (RD) optimization is enabled with all seven inter modes as well as skip, direct and intra modes, and CABAC. Four QPs (i.e. QP=20, 24, 28, and 32) were used for encoding the bitstreams. The experiments were performed on an Intel Xeon X5550 2.67GHz computer with 12GB memory.

\section{A. Rate-Distortion Performances}

Fig. 4 shows the RD performances of different schemes for "Mo2_s1(LBV)". This sequence is a shot of a person turning on a light in a room which causes local brightness variation. From this figure, it can be observed that the coding gains of RWP and FastRWP are about 1dB improvement over NoWP. The gains are still remarkable in comparison with FWP. It is because FWP is a frame-based method and can only use one WP parameter set for each reference frame. Noted that by applying only one WP parameter set to all pixels of whole frame is not efficient for coding a video with LBVs in which brightness variations happen only in partial regions but not in whole frame. Moreover, we can see that the RD curve of FastRWP is close to that of RWP after applying the fast techniques, SRR and FRS, mentioned in Section II.D. 
TABLE I. DETAILS OF VARIOUS VIDEO SEGMENTS OF "MOBisode1" AND "MOBISODE2” USED FOR SIMULATION

\begin{tabular}{|c|c|c|}
\hline $\begin{array}{l}\text { Segment } \\
\text { Name }\end{array}$ & Segment & Characteristics \\
\hline $\begin{array}{c}\text { Mo1_s1 } \\
(\mathrm{GBV})\end{array}$ & $\begin{array}{l}\text { Scene } 1 \text { of Mobisode1 (WVGA, } \\
832 \times 480 \text {, Frame } 178 \text { to } 186)\end{array}$ & $\begin{array}{l}\text { GBV with fade-in-from- } \\
\text { white effect }\end{array}$ \\
\hline $\begin{array}{c}\text { Mo1_s2 } \\
(\mathrm{GBV})\end{array}$ & $\begin{array}{l}\text { Scene } 2 \text { of Mobisode } 1 \text { (WVGA, } \\
832 \times 480 \text {, Frame } 230 \text { to } 249 \text { ) }\end{array}$ & $\begin{array}{l}\text { GBV with fade-in-from- } \\
\text { white effect }\end{array}$ \\
\hline $\begin{array}{l}\text { Mo1_s3 } \\
(\mathrm{LBV})\end{array}$ & $\begin{array}{l}\text { Scene } 3 \text { of Mobisodel (WVGA, } \\
832 \times 480 \text {, Frame } 0 \text { to } 29)\end{array}$ & $\begin{array}{l}\text { LBV with a house at the } \\
\text { centre }\end{array}$ \\
\hline $\begin{array}{l}\mathrm{Mo} 2 \mathrm{~s} 1 \\
(\mathrm{LBV})\end{array}$ & $\begin{array}{l}\text { Scene } 1 \text { of Mobisode } 2 \text { (WVGA, } \\
832 \times 480 \text {, Frame } 42 \text { to } 51 \text { ) }\end{array}$ & $\begin{array}{l}\text { LBV with a person turning } \\
\text { on a light in a room }\end{array}$ \\
\hline $\begin{array}{c}\text { Mo2_s2 } \\
(\mathrm{LBV})\end{array}$ & $\begin{array}{l}\text { Scene } 2 \text { of Mobisode } 2 \text { (WVGA, } \\
832 \times 480 \text {, Frame } 288 \text { to } 299 \text { ) }\end{array}$ & LBV with a door closing \\
\hline
\end{tabular}

Table II shows the experimental results for sequences with various brightness variations using the Bjontegaard delta bitrate (BD-Bitrate) [9] in percentage as well as the relative encoding time compared to NoWP. For RD comparison, from this table, RWP obtains about $12.7 \%$ bitrate reduction in average over NoWP. It is obvious that RWP can overwhelmingly outperform both NoWP and FWP for coding the scenes with LBVs. FastRWP can still obtain $10.1 \%$ bitrate reduction in average compared with NoWP. In the meantime, our schemes, RWP and FastRWP, can still obtain similar performances as the conventional H.264/AVC frame-based scheme, FWP, when coding the scenes with GBVs.

\section{B. Complexity Analysis}

From Table II, it can be seen that the encoding complexity of RWP is 5.2 times that of NoWP. It can be explained that by using the MRF-ME framework of H.264/AVC with five regions including the region without any brightness variation, the number of reference frames is five in total for each forward and backward prediction. Also, the computation of $\mathrm{SAD}$ in (1) for each search point is required to multiply $W$ and add the offset $O$ on-the-fly. This is also the reason that FWP requires the encoding time about 1.2 times that of NoWP. With fast techniques such as SRR and FRS applied into RWP, FastRWP only needs 2.5 times that of NoWP which is half of the encoding time in average compared with RWP.

\section{CONCLUSIONS}

In this paper, a region-based WP scheme is proposed to encode LBV scenes. First, different regions are grouped in accordance with the degree of uniformity in its brightness variation. Second, the precise region-based WP parameter sets are estimated based on the partition. Finally, the framework of MRF-ME is adopted to encode the multiple WP parameter sets without explicitly coding the information of regions. The H.264 framework can then support WP parameter adaptation at a region level. Results show that the proposed RWP can improve the coding performance for scenes with LBV. In addition, we further incorporate fast techniques, SRR and FRS, with our RWP to reduce the number of references for motion estimation. Experimental results further demonstrate that the proposed fast RWP scheme can improve the coding speed while still maintaining the coding performance for LBV scenes.

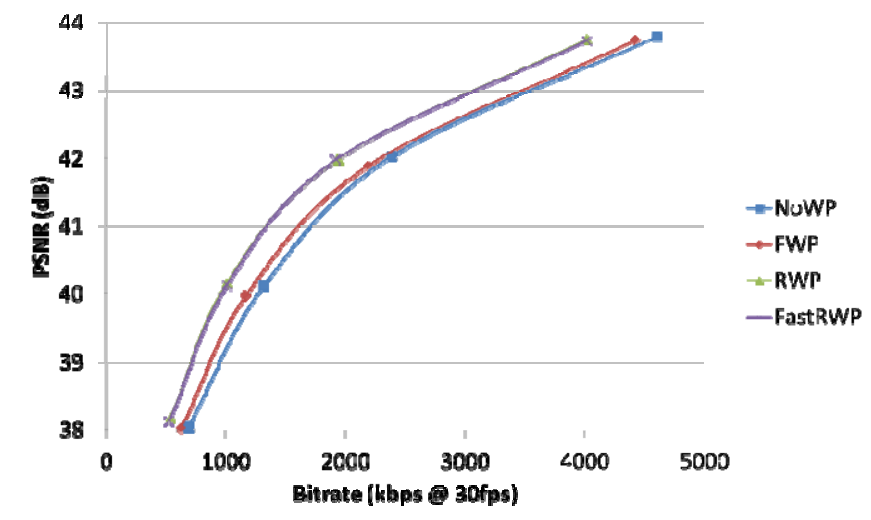

Figure 4. RD performances of different approaches for "Mo2_s1 (LBV)".

TABLE II. BD-BitRATE In PERCENTAGE AND RELATIVE ENCODING TIME OF VARIOUS SCHEMES COMPARED TO NOWP

\begin{tabular}{|c|c|c|c|c|c|c|}
\hline \multirow{2}{*}{$\begin{array}{l}\text { Segment } \\
\text { Name }\end{array}$} & \multicolumn{2}{|c|}{ FWP } & \multicolumn{2}{|l|}{ RWP } & \multicolumn{2}{|c|}{ FastRWP } \\
\hline & BD-Bitrate & Time & BD-Bitrate & Time & BD-Bitrate & Time \\
\hline $\begin{array}{c}\text { Mo1_s1 } \\
(\mathrm{GBV})\end{array}$ & -6.874 & 1.423 & -5.502 & 4.742 & -4.750 & 2.946 \\
\hline $\begin{array}{c}\text { Mo1_s2 } \\
(\mathrm{GBV})\end{array}$ & -26.809 & 0.749 & -31.466 & 3.989 & -22.671 & 2.392 \\
\hline $\begin{array}{c}\text { Mo1_s3 } \\
(\mathrm{LBV})\end{array}$ & -2.118 & 1.343 & -4.867 & 7.093 & -2.510 & 2.581 \\
\hline $\begin{array}{c}\mathrm{Mo} 2 \text { s1 } \\
(\mathrm{LBV})\end{array}$ & -5.565 & 1.199 & -20.699 & 4.379 & -20.325 & 2.577 \\
\hline $\begin{array}{c}\text { Mo2_s2 } \\
(\mathrm{LBV})\end{array}$ & 21.158 & 1.407 & -0.869 & 5.883 & -0.280 & 2.349 \\
\hline Average & -4.042 & 1.224 & -12.681 & 5.217 & -10.107 & 2.569 \\
\hline
\end{tabular}

\section{REFERENCES}

[1] ITU-T Recommendation H.264, "Advanced video coding for generic audiovisual services", March 2010.

[2] J. Boyce, "Weighted prediction in the H.264/MPEG AVC video coding standard," in Proc. IEEE Int. Symp. Circuits Syst., vol. 3, pp. 789-792, Vancouver, Canada, May 2004.

[3] H. Aoki, and Y. Miyamoto, "An H.264 weighted prediction parameter estimation method for fade effects in video scenes," in Proc. IEEE Int. Conf. Image Process., pp. 2112-2115, San Diego, U.S.A., Oct. 2008.

[4] S. H. Kim, and R. H. Park, "Fast local motion-compensated algorithm for video sequence with brightness variations," IEEE Trans. Circuits Syst. Video Technol., vol. 13, no. 4, pp. 289-299, Apr. 2003.

[5] P. Yin, A. M. Tourapis, and J. Boyce, "Localized weighted prediction for video coding," in Proc. IEEE Int. Symp. Circuits Syst., vol. 5, pp. 4365-4368, May 2005, Kobe, Japan.

[6] A. Leontaris and A. M. Tourapis, "Weighted prediction methods for improved motion compensation," in Proc. IEEE Int. Conf. Image Process., pp.1029-1032, Cairo, Egypt, Nov. 2009.

[7] S. H. Tsang, Y. L. Chan, and W. C. Siu, "Multiple weighted prediction models for video coding with brightness variations," IET Image Processing, vol.6, no.4, pp.434-443, June 2012.

[8] (2012) JVT H.264 Reference Software JM18.4. [Online]. Available: http://iphome.hhi.de/suehring/tml/download/.

[9] G. Bjontegaard, "Calculation of average PSNR differences between RD-curves," Document VCEG-M33, ITU-T Q6/SG16, Austin, Texas, U.S.A., Apr. 2001 